\title{
TTR
}

Traduction, terminologie, rédaction

\section{De compte-fil à garde-fou : la lecture du traducteur de poésie}

\section{Madeleine Stratford}

Volume 27, numéro 1, 1er semestre 2014

Lecture et traduction

Reading and Translation

URI : https://id.erudit.org/iderudit/1037119ar

DOI : https://doi.org/10.7202/1037119ar

Aller au sommaire du numéro

\section{Éditeur(s)}

Association canadienne de traductologie

ISSN

0835-8443 (imprimé)

1708-2188 (numérique)

Découvrir la revue

Citer cet article

Stratford, M. (2014). De compte-fil à garde-fou : la lecture du traducteur de poésie. TTR, 27(1),67-93. https://doi.org/10.7202/1037119ar

\section{Résumé de l'article}

Plusieurs représentants de la tradition herméneutique, dont Umberto Eco, Paul Ricoeur et George Steiner, considèrent le texte littéraire inachevé sans le concours du lecteur, lequel est cependant tenu d'en respecter la cohérence interne. Dans un tel contexte, l'affirmation selon laquelle le traducteur serait d'abord un lecteur constitue une évidence qui recèle, en soi, de multiples implications. Dans cet article, nous verrons d'abord comment l'oeuvre littéraire conditionne la lecture et, par conséquent, la traduction pour nous pencher ensuite sur la tension que présuppose la lecture d'un poème à traduire. Pour ce faire, nous comparerons cinq modèles décrivant le processus de traduction poétique des théoriciens suivants : Robert De Beaugrande, Andrei Banta\&scedil;, Francis R. Jones, Christopher Millis et Robert Bly. Ceux-ci s'inscrivent, selon nous, dans la lignée herméneutique en ceci qu'ils préconisent une lecture attentive du poème source, mais cherchent à en contrebalancer les effets par une révision obligatoire minimisant l'apport subjectif du traducteur. L'analyse de ces modèles permettra de constater l'importance réelle de la lecture dans la tâche du traducteur de poésie. 


\title{
De compte-fil à garde-fou : la lecture du traducteur de poésie
}

\author{
Madeleine Stratford \\ Université du Québec en Outaouais
}

\begin{abstract}
Résumé
Plusieurs représentants de la tradition herméneutique, dont Umberto Eco, Paul Ricœur et George Steiner, considèrent le texte littéraire inachevé sans le concours du lecteur, lequel est cependant tenu d'en respecter la cohérence interne. Dans un tel contexte, l'affirmation selon laquelle le traducteur serait d'abord un lecteur constitue une évidence qui recèle, en soi, de multiples implications. Dans cet article, nous verrons d'abord comment l'œuvre littéraire conditionne la lecture et, par conséquent, la traduction pour nous pencher ensuite sur la tension que présuppose la lecture d'un poème à traduire. Pour ce faire, nous comparerons cinq modèles décrivant le processus de traduction poétique des théoriciens suivants: Robert De Beaugrande, Andrei Bantaş, Francis R. Jones, Christopher Millis et Robert Bly. Ceux-ci s'inscrivent, selon nous, dans la lignée herméneutique en ceci qu'ils préconisent une lecture attentive du poème source, mais cherchent à en contrebalancer les effets par une révision obligatoire minimisant l'apport subjectif du traducteur. L'analyse de ces modèles permettra de constater l'importance réelle de la lecture dans la tâche du traducteur de poésie.
\end{abstract}

Mots-clés: herméneutique, lecture, processus de traduction, traducteur, poésie

\begin{abstract}
Many representatives of the hermeneutic tradition, including Umberto Eco, Paul Ricoeur and George Steiner, believe that a literary text is incomplete without the collaboration of the reader, and expect this reader to respect the text's internal coherence. In this context, it seems obvious that a translator is first and foremost a reader, yet this apparently simple observation has many implications. This paper will first show how the literary nature of a text influences the act of reading and of translating it, and then focus on the tension involved in reading a poem for the purpose of translating it. Five models of the poetry translation process will be compared: those of Robert De Beaugrande, Andrei Bantaş, Francis R. Jones, Christopher Millis and Robert Bly. These scholars adopt a hermeneutic approach insofar as they base the translation process on a close reading of the source
\end{abstract}


poem, yet seek to counterbalance its effects by including a necessary stage of revision, minimizing the translator's subjective intervention. The analysis of these models will show what part the act of reading really plays within the process of translating a poem.

Keywords: hermeneutics, reading process, translation process, translator, poetry

\section{Introduction}

Plusieurs théoriciens croient que l'œuvre d'art littéraire est construite dans un matériau poreux, dont les «trous » demandent à être remplis par un lecteur. Entre autres, Umberto Eco postule que le texte constitue «un mécanisme paresseux [...] qui vit sur la plusvalue de sens qui y est introduite par le destinataire» (1985, p. 63). Pour Paul Ricœur, la réception de l'œuvre contribue à parachever «la destinée du texte» (1986, p. 159). Selon George Steiner, le sens profond d'un texte «is at all times the potential sum of individual adaptations»(1992, p. 206). Néanmoins, ils précisent tous les trois que la porosité du texte littéraire est à la fois permissive et contraignante: elle admet plusieurs conjectures plausibles, mais rejette celles qui seraient absurdes. Ainsi, tout interprète, y compris le traducteur, lorsqu'il contribue à reconstruire le texte par sa lecture, est tenu selon eux d'en respecter la cohérence interne, de faire l'équilibre entre la créativité de l'auteur et la sienne propre.

Dans un tel contexte, l'affirmation selon laquelle «the translator must be a reader before becoming a writer »(Zhang,1997, p. 237) est une évidence qui recèle de multiples conséquences. En effet, en se penchant sur la nature de l'œuvre littéraire, le traducteur constate vite que la lecture et la «reproduction» de cette dernière constituent des opérations complexes. L'acte de traduction luimême diffère de la simple lecture en ce qu'il aboutit à une «réécriture»: le traducteur ne se limite pas à recevoir un texte et à le comprendre; il doit également le recréer. Voilà probablement pourquoi Fernand Verhesen déclare que le traducteur de poésie ne devrait être que «prudemment herméneute» et «temporairement philologue», car il devra ultimement devenir "poète à son tour » (2003, p. 19-20).

Dans le présent article, nous accorderons une attention particulière aux types de lecture mis en œuvre lors de la traduction d'un poème. Dans un premier temps, nous exposerons en quoi toute œuvre littéraire conditionne la lecture et, par conséquent, 
la traduction en nous basant notamment sur les propos d'Eco, de Ricœur et de Steiner. Ensuite, nous comparerons cinq modèles décrivant d'un point de vue plus théorique qu'empirique le processus de traduction de textes poétiques proprement dit: celui de Robert De Beaugrande, d'Andrei Bantaş, de Francis R. Jones, de Christopher Millis et de Robert Bly. Les cinq modèles s'inscrivent dans la lignée herméneutique d'Eco, de Ricœur et de Steiner en ceci qu'ils incluent, explicitement ou implicitement, l'acte de lecture/interprétation du poème source, tout en cherchant à en contrebalancer les effets en proposant une ou plusieurs étapes de relecture ou révision obligatoire visant à minimiser l'apport subjectif $\mathrm{du}$ traducteur. Nous proposons donc que chez ces traductologues, la lecture, dans la tâche du traducteur de poésie, se fait tantôt compte-fil permettant de percevoir l'ensemble des caractéristiques du texte source, tantôt garde-fou empêchant de tomber dans une adaptation qui séloignerait trop du style et de l'effet originaux. Cette analyse comparative des différents modèles nous permettra de relativiser et de redéfinir le rôle de la lecture dans la tâche du traducteur de poésie, qui s'approche selon nous davantage de celui de l'arrangeur que de l'interprète d'une pièce musicale.

\section{Umberto Eco : ouverture de l'œuvre et "machine esthétique»}

Selon Eco, un texte serait par définition «inachevé» sans le concours d'un lecteur $(1985$, p. 64$)$ : à son avis, un auteur, consciemment ou non, laisse toujours dans son texte des «trous" à remplir, décrivant le texte comme un «tissu de non-dit». Pour lui, «c'est précisément ce non-dit qui doit être actualisé au niveau de l'actualisation du contenu» (ibid., p. 65). En résumé, tout texte littéraire lui apparaît comme un "mécanisme paresseux», qui non seulement désire qu'un lecteur «l'aide à fonctionner», mais qui incite ce dernier à le faire (ibid., p. 66-67). Il postule que l'existence de tout texte littéraire repose sur cette «ouverture», qu'il croit être «la condition même de la jouissance esthétique» (ibid., p. 59).

Cependant, précise-t-il, bien qu'une œuvre littéraire soit jusqu'à un certain point "ouverte», elle forme aussi un plan guidant le lecteur dans son entreprise de compréhension. En effet, l'auteur ouvre selon lui des portes au lecteur, mais en ferme aussi d'autres, de façon à lui laisser juste ce qu'il faut de place pour qu'il complète le texte sans le composer en entier. Pour Eco, une œuvre 
littéraire devrait donc être tout juste assez malléable pour qu'un lecteur puisse la façonner à sa manière, tout en étant assez solide pour ne pas perdre son individualité, son originalité propre, au cours du processus (1972, p. 141). Le lecteur que décrit Eco est donc tiraillé entre deux pulsions créatrices, celle de l'auteur et la sienne propre, dans un mouvement de va-et-vient entre devoir de fidélité à l'original et liberté d'interprétation créatrice qui n'est pas sans rappeler la tension qui s'opère lors de l'acte traductif.

Il ressort des propos d'Eco que, si bien intentionnée qu'elle soit, toute lecture, parce qu'elle est effectuée par un lecteur empirique, comporte une marge d'erreur. Face à ce danger d'équivoque, Eco postule que seule l' «intentio operis» est souveraine, car il s'agit d'une source «transparente» qui «réfute toute interprétation insoutenable» (1996, p. 71-72). Certes, il est conscient que cette "intention du texte", selon ses mots (mais il me semble que Ricœur en parle aussi) n'existe pas à l'état pur, mais «seulement en tant que résultat d'une conjecture de la part du lecteur» empirique (ibid., p. 58). En théorie, il y aurait donc presque autant d'interprétations possibles que de lecteurs potentiels. Toutefois, bien qu'Eco admette qu'un texte puisse être interprété d'un nombre considérable de manières différentes, il est convaincu que ce dernier refuse de se laisser interpréter n’importe comment (1992, p. 130 et 1996, p. 130-131). En dépit de sa conscience de la très grande difficulté de savoir quelle interprétation est la "meilleure», il croit que le contexte permet toujours d'identifier celles qui sont fausses ou absurdes (1992, p. $130 ; 1996$, p. 47).

Or, repérer le contexte peut s'avérer particulièrement difficile en poésie contemporaine, où, comme l'explique RoseMarie François, ce dernier «est mouvant, immense ou absent. Le mot est là, prégnant de tous ses sens, comme irradié de sens » (1999, p. 76). Robert De Beaugrande déclare pour sa part que la poésie est en soi définie par son intense concentration, qui présuppose un certain degré d'ambiguïté («lack of explicitness ») (1978, p. 30). Ainsi, il arrive fréquemment que le poème ne fournisse aucun indice clair permettant de choisir une signification plus plausible qu'une autre (ibid., p. 39). Le traducteur de poésie semble donc être voué à lire sur la corde raide, risquant toujours de tomber dans l'erreur. 
Peut-être pour cette raison, Eco ajoute-t-il un dernier bémol: même si le contexte permet de valider certaines interprétations (1996, p. 57), chacune demeure selon lui quand même toujours un «pari» (2001, p. 17). En somme, l'interprétation du traducteur, comme celle de tout lecteur, reste subjective, même lorsqu'elle est bien documentée, soutenue par le contexte historico-culturel: "It is just the outcome of an interpretative inference that can or cannot be shared by other readers.» (ibid.,p. 16). À l'image de tous les autres lecteurs empiriques, le traducteur littéraire "parie» donc lui aussi sur le sens du texte sans jamais pouvoir être parfaitement sûr d'avoir gagné ou perdu sa mise (ibid., p. 17).

\section{Paul Ricœur: la polysémie de l'œuvre et l'autonomie du discours}

Déjà avant Eco, Ricœur affirmait qu'un texte littéraire nécessite la participation active d'un lecteur, grâce auquel «s'achève la destinée du texte» (1986, p. 159). En plus de reconnaitre lui aussi l'ouverture propre à la structure du texte, Ricœur souligne l'importance de la polysémie intrinsèque du médium dans lequel il est rédigé: le langage. En effet, explique-t-il, tout texte est fatalement «ambigu» parce que les mots qui le composent sont de nature "polysémique». Selon lui, cette polysémie représente en quelque sorte un mal pour un bien, source d'ambiguité, certes, mais aussi de grande richesse sémantique (1991, p. 449). Or, le fait de devoir choisir entre différentes significations comporte nécessairement un certain risque d'équivoque (ibid., p. 72). Laurence Bougault explique à ce sujet que la poésie contemporaine «fait» toujours sens, même lorsqu'à première vue «elle semble jouer souvent à la limite du non-sens ou au contraire de l'excès de sens. [...] En partant du postulat quelle a malgré tout au moins un sens, on est vite contraint de constater que si l'on veut lui conserver sa spécificité, il faut bien souvent en admettre plusieurs» (2005, p. 439).

En fait, même si Ricœur admet que l'acte de lecture dévoile un excès de signification (1991, p. 401), il croit néanmoins que le but premier de la lecture consiste à canaliser ce surplus d'information en redonnant aux «mots polysémiques» une certaine «intention d'univocité» (1986, p. 77). De Beaugrande va dans le même sens lorsqu'il déclare que, malgré le fait que la polyvalence d'un texte littéraire soit souvent intentionnelle, «most readers feel some need to proceed on the basis of single readings rather than multiple » 
(1978, p. 44). Toutefois, la recherche d'une lecture unique peut se révéler particulièrement problématique pour le traducteur de poésie, qui s'attaque à des textes dont la tâche suprême consiste précisément, selon Ricœur, à faire en sorte que les mots empruntent le plus de significations possible (1991, p. 449). Pour le traducteur de poésie, le véritable défi ne consiste donc pas nécessairement à déceler tous les sens possibles d'un segment donné, mais à arriver à les transposer en langue cible, laquelle ne permet pas toujours de reproduire la polysémie initiale dans toute sa richesse.

Nombre de traductologues sont d'ailleurs d'un avis similaire. La majorité d'entre eux avancent que la tâche du traducteur est de rendre la polysémie du poème, sans toutefois l'accentuer: "Translation should neither increase the difficulties of understanding the poem [...] nor facilitate its comprehension through over-simplification» (Bantaş, 1989, p. 166). Ainsi Françoise Wuilmart estime-t-elle que l'on devrait idéalement «restituer toute la polyvalence du texte original» (1999, p. 214), alors que Rose-Marie François écrit que «la polysémie et l'équivoque du poème doivent se retrouver aussi "ouvertes" dans la traduction» $(1999$, p. 76). De même, selon Fernand Verhesen, "[1]'un des défis majeurs de la traduction de poèmes est d'offrir un éventail de lectures semblables à celui de l'original» (2003, p. 25). Clive Scott va plus loin et fait valoir que le traducteur doit non seulement conserver les ambiguïtés du poème d'origine, mais ne pas hésiter à en rajouter de nouvelles, rendant ainsi le texte encore plus «incompréhensible» $(2006$, p. 106). On peut déduire de cette logique que la tâche du traducteur de poésie comporterait deux mouvements antithétiques: en tant que lecteur, un traducteur cherchera une lecture vectorielle dans cette richesse signifiante, alors que comme «réécrivain» il tentera de rendre sa traduction aussi riche de significations (ou plus riche encore) que l'original.

Outre la nature polysémique du texte, Ricœur croit que sa condition écrite contribue à justifier et même à exiger l'implication du lecteur. Il juge que le discours littéraire échappe partiellement à son créateur originel dès qu'il se retrouve sur la page. Selon lui, les paroles écrites se détachent de l'auteur et acquièrent une vie propre, devenant pour ainsi dire "autonomes». Absent lors de la lecture de son texte, l'auteur n'est plus en mesure d'expliquer lui-même sa signification originelle. Dans l'interaction entre le texte et le lecteur, l'auteur participe indirectement, en différé, et 
ne maîtrise donc pas les réactions de son interlocuteur. De fait, lorsqu'il lit, le lecteur n'échange pas directement avec l'auteur, si bien que «ce n'est pas un cas de dialogue» (Ricœur, 1986, p. 139). En fait, l'œuvre échappe tellement à son auteur qu'elle peut être lue par un public radicalement différent de celui qu'il avait envisagé. Aussi Ricœur voit-il dans le discours écrit (en opposition au discours oral) «une triple autonomie sémantique: par rapport à l'intention du locuteur, à la réception par l'auditoire primitif, aux circonstances économiques, sociales, culturelles de sa production" (ibid., p. 31).

Ces affirmations de Ricœur laissent supposer que ni le lecteur ni le traducteur n'ont à chercher les explications de l'auteur et encore moins son approbation. D'ailleurs, Érik Vigneault opine qu'il ne faut pas confondre l'intention de l'auteur avec l'intention du texte, qui constitue «une manière de vouloir-dire qui résulte de la somme des éléments signifiants du texte» lui-même (1999, p. 174) (Ricœur lui-même fait cette distinction). En somme, le lecteur et le traducteur ne disposent que d'une source valable pour appuyer leur interprétation: le texte lui-même, puisque ce qu'il dit «importe davantage que ce que l'auteur a voulu dire » (Ricœur, 1986, p. 187) ou que ce que les lecteurs de l'original ont bien voulu y comprendre. Dans un tel contexte, l'effet que le traducteur de poésie tentera de reproduire sera probablement celui que le texte a eu sur lui, le seul effet qu'il connaît de l'intérieur. Comme l'explique Verhesen: «Le traducteur ne dispose ni des effets d'un poème et moins encore de ses causes [...]. Il ne dispose que d'un texte, en tout et pour tout, à décrypter en sa profondeur immanente» (2003, p. 12).

Certes, Ricœur admet que toute œuvre ressemble forcément à son auteur: "Parce que le style est un travail qui individue, c'est-à-dire qui produit de l'individuel, il désigne également, rétroactivement, son auteur» (1986, p. 109). En donnant une forme à un contenu, l'auteur laisse des traces de son passage dans le langage de l'œuvre: un vocabulaire, un ton, un rythme particuliers qui constituent sa griffe personnelle. D'une certaine manière, l'ombre de l'écrivain plane sur son texte: même s'il est impossible de retrouver l'intention initiale de l'écrivain, celle-ci y demeure en toile de fond et comme tissée à même le matériau du texte. Bien que l'auteur se taise lorsqu'il dépose sa plume, ce sont pourtant bien ses mots à lui qui font écho durant la lecture 
et qui, par conséquent, couleront dans la plume du traducteur. En fait, lorsque Ricœur conseille aux lecteurs de considérer «l'auteur comme mort et le livre comme posthume» (ibid., p.139), il n'implique pas que l'auteur n'existe pas, mais plutôt qu'il n'est là que textuellement, et non pas physiquement.

En réalité, si l'auteur est présent, c'est à même le tissu du texte, dans la façon dont il s'exprime. L'usage personnalisé qu'un poète fait de sa langue est, selon Roger Caillois, précisément «ce qui permet de l'identifier et qui crée $s a$ poésie, qui empêche qu'on la confonde avec celle de quelqu'un d'autre» (1978, p. 311). Si l'on tient pour acquis que le traducteur de poésie maîtrise la langue de départ, il ne coule pas toujours de source qu'il connaisse bien la parole du poète. Un poème à traduire, précise André Davoust, n'est pas isolé, mais fait plutôt partie d'une constellation particulière, d'une «dynamique intertextuelle» qui demande au traducteur de «recense[r] [1] es particularités stylistiques récurrentes de l'auteur» (1994, p. 116). De Beaugrande recommande lui aussi une étude «systématique» des tics linguistiques du poète à traduire (1978, p. 36-37). Toutefois, Verhesen considère «infiniment plus difficile» pour le traducteur poétique «de se mouvoir dans la langue spécifique, et davantage encore dans le langage propre d'un poète particulier» (2003, p. 9). Poussé à l'extrême, ce postulat impliquerait qu'il faille «développer pour chaque poète [...] une théorie de la traduction» (Kayra, 1998, p. 3).

Le rôle premier du lecteur empirique et, par conséquent, celui du traducteur, consiste selon Ricœur à se laisser guider par le texte, en allant "dans son sens" de façon à découvrir son «intention" ou sa "visée» (1986, p.156). Cette intention du texte ne saute toutefois pas aux yeux puisqu'elle est «autre chose que la somme des significations individuelles des phrases individuelles» (ibid., p. 201). L'œuvre polysémique doit être abordée non pas en pièces détachées, mais en bloc, car elle résulte d'un «processus cumulatif, holistique» (ibid.; Ricoeur, 1991, p. 312). Voilà probablement pourquoi Ricœur considère lui aussi que le mouvement traductif devrait toujours partir du contexte pour finir par le mot, allant du plus large au plus étroit, un peu comme si le texte passait par un entonnoir: "s'imprégnant par de vastes lectures de l'esprit d'une culture, le traducteur redescend du texte, à la phrase, au mot» (2004, p. 56). Or, c'est justement ce que De Beaugrande veut dire lorsqu'il écrit qu'en traduction poétique, «the word cannot be the 
unit of translation, but only the text in communication» (1978, p. 91). Il conseille d'ailleurs lui aussi de procéder «à l'envers» ne cherchant l'équivalence lexicale qu'une fois le contexte établi.

Paradoxalement, il est impossible d'assembler des phrases ou des mots sans les examiner de près. Lecteurs et traducteurs se retrouvent en quelque sorte dans une impasse, puisque «la reconstitution d'un texte en tant que tout offre [...] un caractère circulaire en ce sens que la présupposition d'une certaine sorte de tout est impliquée dans la reconnaissance des parties» (Ricœur, 1986, p. 200). Or, contrairement à quelqu'un qui fait un cassetête, ni le lecteur ni le traducteur n'ont accès à l'image originale, mais ils doivent plutôt s'en forger une eux-mêmes. Ainsi, explique De Beaugrande au sujet de la traduction d'un poème, "[t]he basis of the act of translation is not the original text, but rather the representation of the text that is eventually generated in the translator's mind» $(1978$, p. 25$)$. Lorsqu'il fait ses choix de compréhension, le traducteur procède à partir de ses lectures passées, de son expérience de vie, de sa personnalité, de sa culture et de sa langue. Il introduit donc beaucoup de lui-même dans sa lecture. Conscient du caractère subjectif de toute interprétation, Ricœur déclare que «c'est toujours quelqu'un qui reçoit, fait sien, s'approprie le sens» (1986, p. 168).

Loin d'être toujours identique, l'intention du texte prendra ainsi inévitablement la couleur de la perception qu'en a son lecteur. Mais il y a plus: un mouvement "d'appropriation» serait, selon Ricœur, non seulement inévitable, mais souhaitable. En effet, cette volonté de "rapprocher» et de «rendre contemporain et semblable» à soi constitue, selon lui, l'un des buts fondamentaux de toute herméneutique (Ricœur, 1986, p.153 et 1991, p. 89). Malgré lui, le traducteur, tout comme le lecteur, est en quelque sorte condamné à l'appropriation. Cela explique probablement pourquoi chaque traduction d'un même ouvrage est si différente, même si elle vise à être équivalente à l'original: "Cette équivalence ne peut être que cherchée, travaillée, présumée. Et la seule façon de critiquer une traduction [...], c'est d'en proposer une autre présumée, prétendue, meilleure ou différente» (Ricœur, 2004, p. 40). Ainsi, aucune traduction ne suffit à reproduire à elle seule le potentiel significatif d'un poème: «it takes an unending series of translations, from the crib to the verse rendering, to even begin to approximate the poem, which remains an asymptote they'll never 
quite reach»(Folkart, 1999, p. 32). Clive Scott va dans le même sens en affirmant qu'une version existante d'un poème n'est en fait que la réalisation concrète d'une seule des multiples possibilités de traduction (2006, p. 110).

Toutefois, Ricœur précise que la plausibilité de plusieurs interprétations ne garantit pas que toutes soient aussi valides. Comme Eco, il estime qu'un lecteur ne peut faire dire au texte ce qu'il ne dit pas, sous peine d'en détruire la cohérence (Ricœur, 1991, p. 496). Ainsi Ricœur conçoit-il l'œuvre littéraire comme "un champ limité de constructions possibles», même lorsque leur nombre est si grand qu'on ne peut les répertorier toutes (1986, p. 202; nous soulignons). En fait, l'objet du texte ne change jamais: à chaque lecture, il reste imprimé dans les mêmes caractères et sur le même papier et ses phrases sont agencées de la même façon. Le texte présenterait donc au lecteur une série limitée d'options et chaque choix interprétatif conditionnerait le suivant, faisant ainsi bifurquer le texte dans un sens différent, mais déjà prévu dans sa structure. Pour Ricœur, comme pour Eco, c'est le contexte qui servira toujours de guide et empêchera le lecteur et le traducteur de s'égarer dans des conjectures qui ne tiennent pas la route (Ricœur, 1991, p. 71 et 2004, p. 47). Cela dit, nous avons vu plus haut que ce «contexte» n'est pas toujours présent en poésie contemporaine, ce qui contribue à relativiser la portée des commentaires de Ricœur et d'Eco pour les traducteurs de ce genre de textes.

\section{George Steiner: la primauté du corps textuel et le parcours herméneutique}

Comme Ricœur, Steiner est d'avis que la nature «imprécise» du langage est à l'origine de son constant besoin d'être interprété: «Even the most literal statement [...] has a hermeneutic dimension. It needs decoding. It means more or less or something other than it says» (1992, p. 295). Steiner croit lui aussi que la littérature en général, et en particulier la poésie, exploite à son maximum cette polysémie: "Multiplicity of meaning, "enclosedness," are the rule rather than the exception» (1978, p. 21). Il s'inscrit donc dans la même lignée qu'Eco lorsqu'il déclare que le sens du texte ne peut exister sans être actualisé par un ou plusieurs lecteurs: «Meaning is at all times the potential sum of individual adaptations» (Steiner, 1992, p. 206). À l'instar d'Eco et de Ricœur, Steiner avance que chaque lecture vise à atteindre une certaine univocité. En effet, 
il considère la recherche d'un sens précis comme l'assise de la compréhension de tout discours, bien qu'il admette l'inaccessibilité d'un véritable sens unique:

Nous devons lire comme si le texte devant nous avait un sens $[\ldots]$ un sens que l'exégèse, le commentaire, la traduction, la paraphrase, le décodage, psychanalytique ou sociologique [ne] pourront jamais épuiser, jamais définir comme total. (Steiner, 1988, p. 61-62)

Le «comme si» de Steiner souligne le caractère hasardeux de la lecture et rappelle ainsi le «pari interprétatif» dont parlait Eco. En fait, il appert que, selon ces théoriciens, lecteurs et traducteurs font toujours «comme si» ils étaient sûrs d'avoir la bonne réponse à l'énigme du texte. Jusqu'à un certain point, s'il veut arriver à produire sa ou ses versions, le traducteur de poésie n’a lui aussi d'autre choix que de faire «comme si».

Si le lecteur ne peut jamais comprendre totalement le texte littéraire, c'est parce qu'il ne peut l'aborder que de façon subjective. Abondant ici dans le même sens qu'Eco et Ricœur, Steiner (1992) postule que l'on ne peut aborder un texte qu'à partir de sa propre expérience, qui diffère par définition d'une personne à l'autre. D'ailleurs, Steiner ajoute que non seulement chaque lecteur, mais aussi chaque lecture produit sa propre interprétation d'un même texte: «each reading, each translation differs, each is undertaken from a distinctive angle of vision» (ibid., p. 30). En effet, il arrive souvent qu'en relisant un livre, un même lecteur y découvre un nouveau sens, différent de celui qu'avait généré sa première lecture. Or, si un poème donne lieu à de nombreuses lectures, il va de soi qu'il puisse inspirer plusieurs traductions distinctes, produites de façon consécutive ou simultanée, publiées ou non, que leur auteur connaisse ou non les versions précédentes. Voilà pourquoi Albert Schneider considère qu'une «traduction de poème nest jamais terminée. L'auteur s'arrête à une certaine solution, c'est tout» (1978, p. 34). Mais le fait qu'un traducteur donné se soit «arrêté à une certaine solution» ne signifie pas pour autant que «ce soit tout», car rien n'empêche un autre traducteur de contribuer lui aussi au processus «jamais terminé» de la traduction du poème. En fait, un même traducteur est susceptible d'interpréter un poème et de le transposer de multiples façons. 
Face à une telle pluralité de points de vue possibles, Steiner juge «indispensable» que le lecteur appuie ses conjectures sur une bonne connaissance de la culture et de la langue du texte; il ajoute même que le fait d'être familier avec l'ensemble de l'œuvre d'un auteur et sa bibliographie est toujours d'un grand secours (1992, p.26). Cette conception expliquerait notamment l'affirmation de Steiner selon laquelle grammaires et dictionnaires n'aident en aucun cas le traducteur littéraire à comprendre un texte, mais que seul le contexte, «in the fullest linguistic-cultural sense, certifies meaning» (ibid., p. 376).

À première vue, on dirait donc que Steiner suggère au lecteur (et au traducteur) de replacer l'œuvre dans son contexte de production pour que sa compréhension soit la plus juste possible: «Nous devons lire comme si l'environnement temporel et la mise en ouvre d'un texte avaient de l'importance» (1988, p. 62). Néanmoins, la présence d'une formulation hypothétique ("comme si») laisse supposer que Steiner n'adhère pas tout à fait à sa propre affirmation. Quelques lignes plus loin, il précise d'ailleurs explicitement la "vulnérabilité» de l'aide apportée par les informations contextuelles (ibid.). Du reste, Steiner est tout aussi convaincu qu'Eco et que Ricœur de la futilité de toute tentative de retrouver l'intention de l'auteur: «l'idée [...] que nous pourrions revenir à ce qu'il voulait nous dire de son propre sujet dans notre compréhension de son texte, est totalement naïve" (ibid., p. 55). Chaque œuvre littéraire posséderait, selon Steiner, une identité propre qui n'est pourtant jamais identique à celle de son créateur. Plus radical encore que Ricœur, qui reconnaît dans le style du texte la signature de l'auteur, Steiner juge qu'à la limite, il est absolument inutile de "connaître le nom du poète pour lire le poème» (ibid., p. 54). Au bout du compte, Steiner suggère qu'un lecteur compétent ne devrait pas chercher à aborder le texte d'une manière "érudite», mais plutôt se fier à son «intuition " personnelle (1992, p. 26).

Steiner postule, à l'image d'Eco et de Ricœur, que le texte constitue la seule source fiable sur laquelle un lecteur puisse baser son interprétation: "Sa priorité est d'essence, de nécessité ontologique et d'auto-suffisance» (1988, p.59). Cette œuvre littéraire, Steiner la décrit comme un tout, une «structure organique et circulaire (self-informing) [...] [qui] rend vulnérable à l'extrême l'analyse d'unités sémantiques ou de moments isolés» 
(2003, p. 211). Selon lui, une lecture véritable reconnaît la «singularité» de l'œuvre littéraire en tant que "présence réelle d'un être signifiant» (Steiner, 1988, p. 62-63). Comme Ricœur, Steiner conseille au lecteur d'aborder le poème dans sa totalité plutôt que dans ses mots isolés, puisque son originalité réside justement dans l'agencement inaltérable des éléments qui le composent. Par ailleurs, Steiner considère quen poésie, le sens se retrouve autant dans la forme du texte que dans son contenu. À son avis, «une forme poétique "extériorise", "joue" (acts out) son sens" (2003, p.195). Dans After Babel, il va même jusqu'à avancer qu'un traducteur devrait tenter de reproduire le corps physique d'un poème avant même de s'attaquer à son sens: «great translation moves by touch, finding the matching shape, the corresponding rugosity even before it looks for counterpart of meaning» (Steiner, 1992, p. 308). Lors de sa lecture, le traducteur que décrit Steiner observe le poème d'abord et avant tout en tant quobjet tangible; il ne cherche pas seulement à comprendre les significations que les mots cachent, mais regarde aussi les mots eux-mêmes, en apprécie les contours et l'agencement. Voilà une étape qui semble cruciale pour le traducteur de poésie contemporaine, dont la forme «libre» ne correspond souvent pas à des modèles préétablis.

Steiner établit un lien explicite entre lecture et traduction lorsqu'il définit cette dernière comme un "parcours herméneutique». Selon lui, ce "parcours» compte quatre étapes qui résument le processus traductif, de la lecture à la rédaction. Dans un premier temps, le traducteur-interprète doit «faire confiance» au texte original. En effet, il doit tenir pour acquis que l'œuvre n'est pas vide de sens, qu'il y a là quelque chose à comprendre: "As he sets out, the translator must gamble ${ }^{1}$ on the coherence, on the symbolic plenitude of the world» (1992, p. 313). Dans un deuxième temps, le traducteur s'«attaque» au texte dans tous les sens du terme; il l'assaille de toutes parts, cherchant à se l'approprier totalement: "Comprehension, as its etymology shows, "comprehends" not only cognitively but by encirclement and ingestion» (ibid., p. 314). Or, tel est pris qui croyait prendre: le texte «importé» de force pénètre les habitudes du traducteur pour les changer. Steiner décrit cette troisième étape comme étant forcément «incorporative » : «No language, no traditional symbolic set or cultural ensemble imports without the

1. Cette idée rappelle le «pari interprétatif» d'Eco. 
risk of being transformed»(ibid., p. 314-315). S’il accepte cette transformation et redonne au texte une partie de ce qu'il lui avait dérobé, le traducteur est arrivé à la quatrième et dernière étape du processus herméneutique. Le traducteur montre son respect pour le texte dans cet acte de "compensation ": "The translator [...] is faithful to his text, makes his response responsible, only when he endeavours to restore the balance of forces, of integral presence, which his appropriative comprehension has disrupted" (ibid., p. 318-319). Quelques siècles avant Steiner, Friedrich von Schlegel reconnaissait déjà l'importance de ce quatrième mouvement de «restitution» lorsqu'il déclarait: "Pour comprendre quelqu'un qui ne se comprend qu'à moitié, il faut d'abord le comprendre à fond et mieux que lui-même, puis à moitié et tout juste comme lui» (Schlegel cité dans Berman, 1984, p. 171).

En somme, cette analyse des propos d'Eco, de Ricœur et de Steiner démontre que l'œuvre d'art littéraire (et peut-être plus encore le poème) est construite dans un matériau poreux, dont les «trous", nous l'avons déjà dit, demandent à être remplis par un lecteur. En effet, ces trois théoriciens estiment que pour comprendre le texte, tout lecteur, y compris le traducteur, doit y entrer, s'y investir et se l'approprier. Comme le lecteur qu'ils décrivent, le traducteur interprète le texte de façon autonome, puisqu'il n'a souvent accès ni à l'auteur réel ni au premier public de l'original. Lecteur actif, perspicace et créatif, le traducteur se laisse guider par son instinct, mais il ne jouit pas d'une liberté sans bornes, car le texte écrit présente une structure à la fois permissive et contraignante qui admet plusieurs conjectures plausibles, tout en rejetant celles qui seraient absurdes. Ainsi, tout interprète, y compris le traducteur cherche-t-il à établir un équilibre entre la créativité de l'auteur et la sienne propre, tout en respectant la cohérence et la cohésion du texte. Voyons à présent comment cette conception herméneutique de la lecture et de la traduction se décline dans les modèles du processus de traduction poétique de Robert De Beaugrande, Andrei Bantaş, Francis R. Jones, Christopher Millis et Robert Bly.

\section{Le processus de la traduction poétique: de la lecture à l'écriture}

Steiner n'est pas le seul théoricien à avoir cherché à décrire de façon systématique la nature du processus de traduction d'un texte littéraire. Après avoir consulté les bases de données MLA 
Bibliography, LLBA et Érudit, où sont répertoriés l'ensemble des articles publiés dans les revues scientifiques spécialisées en littérature et en traductologie, nous avons retenu cinq modèles visant à décrire exclusivement le processus de la traduction de textes poétiques. Dans la section qui suit, nous verrons que les modèles de De Beaugrande, Bantaş, Jones et Bly décrivent un processus analogue qui débute avec la lecture et l'analyse du poème source, passe par l'évaluation des caractéristiques formelles et thématiques ainsi que de leur importance respective par rapport à l'ensemble du poème, puis se termine par la production d'un texte cible qui peut, lui aussi, être considéré comme un poème. Le processus que décrit Millis diffère légèrement des autres en ceci qu'il omet la lecture préalable du poème et commence directement avec la rédaction d'une "première version littérale » (1988, p. 21) $)^{2}$.

S'inspirant du parcours herméneutique de Steiner et des trois catégories de traduction de Roman Jakobson (intralinguale, interlinguale et intersémiotique), De Beaugrande (1978) identifie sept phases qui sont, selon lui, indispensables à la réalisation de la traduction poétique, que le traducteur soit conscient ou non de les avoir franchies. Ces sept étapes peuvent avoir lieu consécutivement ou parallèlement: 1) représentation mentale du texte;2) repérage d'éléments «inhabituels» dans le texte, comparés à de possibles équivalents plus «habituels»; 3) évaluation de la valeur communicative respective des divers segments de texte; 4) interprétation des éléments problématiques à l'aide du contexte et du cotexte; 5) recherche d'équivalents potentiels pour la reconstruction en langue cible de l'ensemble de la représentation mentale construite aux étapes précédentes; 6) comparaison entre la représentation mentale de la traduction résultante et celle du texte d'origine; 7) révision de la traduction (si possible par d'autres lecteurs) dans le but de prévoir les réactions du public cible.

Le processus de traduction poétique d'Andrei Bantaş (1989) comporte pour sa part six étapes: 1) quête d'information sur l'auteur et son œuvre; 2) analyse lexico-sémantique de l'original; 3) analyse grammaticale de l'original; 4a) analyse de l'utilisation de synonymes (mots, syntagmes, expressions idiomatiques, phrases, vers, strophes, rythme, accentuation, rime et tout autre recours

2. Voir l'annexe pour un tableau comparatif des modèles. 
prosodique), suivie de $4 \mathrm{~b}$ ) recherche de synonymes potentiels dans le texte cible; 5) rédaction d'un brouillon de la traduction incorporant les résultats des étapes 1 à 4 , mais sans préoccupation pour la forme du poème; 6) rédaction de la traduction finale en tenant compte de la forme du poème (versification). Bantaş souligne l'importance de l'analyse du traducteur (étapes 1 à 4), selon lui «indispensables» (1989, p. 149).

Bien qu'énoncé en des termes différents, le modèle de Bantaş ressemble à celui de De Beaugrande. D'abord, la première étape de Bantaş, qui concerne la quête d'information contextuelle, s'apparente à la quatrième étape du processus décrit par De Beaugrande, où le «contexte» et le «cotexte» aident le traducteur à interpréter les ambiguïtés du poème. Ensuite, les étapes 2 à $4 \mathrm{a}$ de Bantaş décrivent en détail ce que De Beaugrande rassemble au point 2 sous le «repérage» des caractéristiques linguistiques distinctives du poème. En outre, la cinquième étape du modèle de De Beaugrande, relative à la recherche d'équivalents des caractéristiques repérées, se retrouve aux points 4 et 5 de Bantaş, où sont cherchés des "synonymes" équivalents et où est rédigé un premier brouillon. Enfin, la sixième étape de chacun des modèles concerne dans les deux cas une relecture du poème original et une révision de la traduction pour que l'effet produit soit le plus équivalent possible à celui produit par l'original. En réalité, seule la septième étape du modèle de De Beaugrande, celle où la traduction est révisée par une tierce personne pour qu'elle soit «acceptable» aux yeux du public cible, ne se retrouve pas dans le modèle de Bantaş.

Le modèle proposé par Jones (1989) décrit trois macroétapes principales qui englobent l'ensemble des étapes énumérées précédemment: la compréhension, l'interprétation et la création. La première phase ne touche que le texte source et inclut deux étapes: la ou les lectures et l'analyse des caractéristiques thématiques et formelles qui font de ce texte un poème. Lors de la deuxième phase, le traducteur évalue l'importance des différentes caractéristiques identifiées et la possibilité de les reproduire dans la langue cible. Jones postule que les réseaux de signifiance du texte possèdent une certaine "valence", pouvoir de signifiance dont on peut évaluer la force, ce qui nous permet d'identifier les éléments contribuant le plus au sens et à l'effet du poème. Les caractéristiques de cette "valence» sont multiples et repérables 
à tous les niveaux: sémantique, étymologique, symbolique, syntaxique, sonore, morphologique, stylistique, etc.

Si Jones accorde une certaine importance au cotexte lors de son évaluation, comme De Beaugrande et Bantaş, il ne semble pourtant pas considérer les contextes de production et de réception du poème original. Quant à la troisième phase de Jones, elle s'éloigne des microstructures pour se concentrer à nouveau sur l'ensemble du texte et la recréation d'un effet global acceptable pour les lecteurs cibles, ce qui équivaut globalement aux deux dernières étapes du modèle de De Beaugrande et à la sixième étape de celui de Bantaş.

Dans ses grandes lignes, le processus de traduction poétique que Jones décrit n'apporte donc pas beaucoup plus que les deux autres modèles. Jones se démarque néanmoins par l'importance particulière qu'il accorde à la phase d'interprétation en suggérant cinq stratégies d'équivalences que le traducteur peut adopter: 1) le transfert, où un élément du texte cible est en tous points équivalent à un autre du texte source; 2) la convergence ou la divergence, où un élément du texte cible occupe plus ou moins d'espace sémantique que dans le texte source, mais dont la «valence» demeure constante; 3) l'improvisation, où une des composantes du texte cible differe du texte source, tout en jouant cependant un rôle poétique similaire; 4) l'abandon, où une caractéristique jugée moins importante est délaissée au profit d'une caractéristique jugée plus importante; 5) l'effet d'étrangeté, où un élément de la langue source est reproduit tel quel, sans être traduit. Jones situe ces cinq stratégies dans une approche pédagogique à l'intérieur du processus de traduction, mais elles peuvent aussi, selon nous, servir d'outils méthodologiques permettant d'analyser plusieurs produits de cet acte traductif, c'est-à-dire des traductions existantes, proposition qui a d'ailleurs été mise en pratique (Stratford, 2011).

Comme nous l'avons précisé plus haut, le modèle de Christopher Millis (1988) ne débute pas explicitement avec la lecture du texte source, mais il semble la présupposer. En effet, la première étape, qui concerne la rédaction d'une «première version littérale", implique que le traducteur soit d'abord entré en contact avec le texte une ou plusieurs fois avant de le traduire. Millis souligne l'apport interprétatif du traducteur lorsqu'il écrit que, même lors de l'écriture d'un premier brouillon se voulant «littéral», le traducteur laisse sa trace. Par ailleurs, il est intéressant 
de constater à quel point les deuxième et troisième étapes du modèle de Millis ressemblent à celles de traduction et de révision décrites par De Beaugrande et Jones. En effet, outre la première étape de "version littérale», le modèle de Millis compte, comme ceux de Jones et de De Beaugrande, deux étapes de révision. La première porte sur l'adéquation du texte au style du poète, ou du moins à la perception qu'en a le traducteur. La deuxième étape de révision concerne la correction de la version du traducteur selon des normes littéraires cibles: "In terms of focus, attention has shifted form listening to the [source language] to listening to [the target language]» (Millis, 1988, p. 22).

Le modèle de Robert Bly (1983) a ceci en commun avec celui de Millis qu'il débute avec la production d'un premier brouillon littéral, qui présuppose vraisemblablement une lecture préalable du poème source. Lors de cette étape intuitive, le traducteur se laisse complètement aller. Il retourne ensuite au poème original et en effectue une analyse sémantique détaillée afin de le comprendre à fond et de repérer les «détails problématiques ", idéalement aidé par un locuteur natif de la langue source. Les six étapes suivantes ressemblent aux macro-étapes des autres modèles, mais se divisent en une série de micro-étapes qui donnent chacune lieu à une nouvelle version. La troisième est une étape de «retraduction»: elle consiste à produire une nouvelle version littérale, mais idiomatique, qui rende compte de la lecture approfondie du texte source et se soucie des structures cibles. La quatrième étape parachève la troisième en incorporant dans la nouvelle version des marques d'oralité afin de lui prêter encore plus de naturel. Par la suite, Bly s'attarde à quatre étapes de révision centrées sur la reproduction de la forme (essentiellement sonore) du poème, lesquelles impliquent plusieurs allers-retours entre les étapes de relecture (de l'original et des versions produites) et de retraduction. La cinquième étape est la reproduction du ton; la sixième consiste en a) la recréation de l'effet des patrons sonore et rythmique, impliquant en b) plusieurs lectures à voix haute du poème source; la septième est la révision par un locuteur natif de la langue source pour minimiser les infidélités potentielles; et la huitième est la production de la version finale, qui pourra aussi tenir compte, le cas échéant, des traductions préalablement publiées. Là où le modèle de Bly se démarque le plus est dans les deux dernières étapes de révision, où il donne préséance non pas au lecteur cible, 
mais au lecteur source, et où il tient compte, pour le peaufinage de sa version finale, des traductions déjà parues. Il est aussi le seul qui accorde autant d'importance à l'aspect sonore du poème et à sa reproduction.

En définitive, lordre des étapes et la terminologie utilisés par les auteurs étudiés diffèrent légèrement, mais l'essentiel du contenu s'y retrouve, de la lecture interprétative du poème source à la production de la traduction proprement dite, puis à sa révision. Tous les modèles supposent la production de plusieurs versions. Puisque l'interprétation du texte source et l'analyse de ses constituants forment la base explicite de quatre des cinq modèles étudiés, l'apport subjectif du traducteur apparaît intrinsèque à tout processus de traduction poétique. Même le recours à un locuteur de langue source proposé par Bly ne garantira pas pour autant que la lecture du poème soit plus objective. Non seulement le traducteur fait des choix lors de sa lecture, mais il en fait également lorsque la langue cible lui offre plusieurs possibilités de traductions. Néanmoins, chaque modèle cherche à minimiser ce caractère subjectif dans une dernière étape de révision ou de relecture où sont presque toujours pris en compte tant le degré d'équivalence à l'original que le degré d'acceptabilité de la traduction auprès du public cible.

Certes, les modèles analysés ici sont avant tout le produit d'expériences individuelles, et même si les témoignages partagent des ressemblances indéniables, leur représentation du processus de traduction poétique demeure essentiellement théorique, avec un ton prescriptif sous-jacent. À cet égard, nous partageons l'avis d'Andreas Wittbrodt (1995), qui juge qu'une théorie qui se veut «normative» se discrédite comme théorie au sens strict du terme, puisqu'elle ne repose pas sur l'identification objective de critères. En fait, comme le remarque Jones en 2006, peu d'investigations empiriques ont été effectuées à ce jour sur ce qui se produit dans la «boîte noire» du traducteur de poésie. Dans son article, Jones tente de remédier à ce manque en étudiant empiriquement le processus de traduction poétique: il effectue une série d'entrevues semi-dirigées avec cinq traducteurs chevronnés, puis se soumet luimême à un exercice de traduction avec protocole de verbalisation (think-aloud protocol). On pourra s'interroger sur la représentativité des sujets choisis ainsi que sur la portée générale des conclusions d'une recherche de type traditionnel où l'objet d'étude devient le 
chercheur lui-même. Toutefois, Jones est conscient de ces limites. Quoi qu'il en soit, certaines de ses observations sont intéressantes parce qu'elles confirment les tendances énoncées dans les cinq modèles traités précédemment, ce qui indique que les modèles analysés, même sans adopter une méthodologie propre aux études empiriques, demeurent près de la réalité.

En premier lieu ou dans un premier temps, les cinq traducteurs interviewés par Jones ont dit qu'ils produisaient eux aussi plusieurs «brouillons» (drafts) d'un même poème entre lesquels ils faisaient des pauses (drawer time), et qu'ils recouraient parfois à l'aide d'autres traducteurs (2006, p. 64). Ensuite, les quatre versions produites successivement par Jones aux fins de l'étude recoupent grosso modo les étapes de traduction et de révision que celui-ci mentionnait en 1989: les versions 1 et 2 se concentrent sur la reproduction de microstructures (surtout lexicales, grammaticales et stylistiques dans la version 1 ; surtout formelles dans la version 2), ce qui correspondrait à l'étape 4 de son modèle, tandis que les versions 3 et 4 se préoccupent davantage de la reproduction du poème global en tant que macrostructure, ce qui correspondrait à l'étape $5 \mathrm{du}$ modèle. Le processus de traduction poétique s'apparente à une démarche herméneutique, marquée par la production de versions successives nourries de multiples lectures et relectures du texte source ainsi que de révisions du ou des textes cibles, par le traducteur lui-même et éventuellement par de tierces personnes.

\section{Conclusion}

Il ressort de cette analyse que le traducteur de poésie est effectivement, mais non essentiellement, un lecteur. En fait, c'est probablement parce qu'il prend son rôle de lecteur actif au sérieux que le traducteur de poésie décrit par les théoriciens cités ici lit et relit si souvent le texte source et ses propres versions tout au long du processus. À en juger par les modèles étudiés, celui-ci ne se contente pas d'offrir ce qu'Eco nomme une «interprétation sémantique» de l'œuvre (1992, p. 36). Au contraire, il afficherait plutôt ce qu'Eco qualifierait d'attitude «critique»: passer de la simple interprétation au désir d' «explication» en cherchant à comprendre le comment et le pourquoi de son interprétation du texte (ibid., p. 36). On peut en conclure que le traducteur de poésie lit son texte source à la loupe; qu'il ne se contente pas de recevoir 
ou de vivre l'effet esthétique (comme le ferait un simple lecteur), mais qu'il s'efforce de trouver et d' "apprécier» les «stratégies textuelles » qui en sont à l'origine (Eco, 2001, p. 93-94), de façon à les reproduire de son mieux dans le texte d'arrivée, à titre cette fois de réécrivain, et non plus de lecteur. Lors de cette phase d'écriture, on pourrait dire que la lecture au compte-fil du texte source se transforme en une lecture garde-fou des textes source et cible: elle vise à faire en sorte que le traducteur, dans sa re-création, ne tombe pas dans un excès d'adaptation qui ferait en sorte que les lecteurs cibles ne puissent plus reconnaître le style ou le contenu sources.

Par ailleurs, lorsqu'il est question de poésie, plusieurs aiment à soulever, comme Robert Bly, une analogie entre le vers et les notes, entre les mots et la musique ${ }^{3}$. Selon John Hollander, cette tendance à accorder tant d'importance à l'aspect sonore du poème prendrait sa source dans l'Antiquité, où l'on avait établi une relation d'identité entre musique et poésie (1985, p. 7). Même si de nos jours, explique Hollander, on ne confond plus ces deux arts, la volonté d'identifier la poésie à un médium sonore persiste (ibid., p. 246). Par conséquent, il n'est guère étonnant qu'un lieu commun consiste à comparer le traducteur littéraire à un musicien qui «interprète» un morceau de musique, une métaphore qui n'est pas sans rappeler la comparaison entre lecteur et traducteur. Selon cette conception, le musicien interpréterait pour l'oreille externe, et le traducteur, pour ce que Hans-Georg Gadamer appellerait son «oreille interne» (1982, p. 178), que Jacques Derrida nomme pour sa part la "petite oreille» (1982, p. 180). Comme le musicien lors de son exécution, le traducteur devrait user de tout son talent d'artiste pour capter dans la langue d'arrivée l'esprit de la langue (ou de la musique) originale ainsi que le style de l'auteur (ou du compositeur).

À bien y songer, cette analogie entre la musique et la poésie ou la littérature se rapporte selon nous plus au processus de lecture qu'à la traduction proprement dite et contribue ainsi à perpétuer la confusion entre le rôle du lecteur et le rôle du traducteur.

3. Entre autres, Roland Maisonneuve dit que le traducteur de poésie doit chercher à «recomposer un nouvel équilibre musical» (1978, p. 77); Erol Kayra parle de l'«esthétique» d'un poème, il ne fait allusion qu'à son «caractère sonore» (1998, p. 1); Léon Robel limite même l'appellation «traduction poétique» aux versions qui reproduisent la «structure profonde phono-sémantique de l'original» (1973, p. 62). 
Contrairement au lecteur ou au musicien, qui n'ont pas besoin de "concrétiser» leur lecture en un nouveau texte ou une nouvelle partition, le traducteur de poésie doit écrire, car la rédaction d'un poème constitue justement le but ultime de sa lecture. Il ne traduit donc pas que pour «l'oreille» (petite ou grande, interne ou externe), mais aussi pour l'œil, et la fixation sur papier d'un autre texte diffère d'une performance en direct, en ceci que «les écrits restent», comme le dit le proverbe.

Par conséquent, nous croyons nécessaire de modifier la traditionnelle métaphore musicale. Plutôt que d'identifier le traducteur de poésie exclusivement à l'interprète (ou au lecteur), nous proposons qu'à première vue, son rôle ressemble davantage, du moins dans sa phase d'écriture, à celui de «l'arrangeur musical», un compositeur qui lit puis transcrit une partition pour un ou plusieurs instruments autres que celui pour lequel la pièce avait été composée. En effet, comme l'explique le compositeur français François Nicolas, la transcription «transport[e] une musique dans une autre formation instrumentale», s'efforçant pour que l'«instrument apprenne lui-même à supporter une musique qui n'a pas a priori été conçue pour lui» (2000, p. 51-52). D'ailleurs, l'arrangeur «transcrit» souvent, comme le traducteur, directement à partir de sa lecture de la partition originale, sans l'avoir nécessairement entendue.

Certains diront que l'idée de comparer le traducteur à un arrangeur est plus ou moins novatrice. En effet, le traductologue Jeffrey M. Green a déjà suggéré de comparer le traducteur à l'arrangeur en raison de leur qualité d'artiste et leur créativité indéniables, mais aussi de leur "subordination» à l'auteur original (2001, p. 30). De façon similaire, Allan Turner a déjà proposé que les traductions, comme les transcriptions «expertes», peuvent atteindre une qualité artistique comparable à l'œuvre originale, et même révéler (volontairement ou non) l'intention initiale de son auteur: «[...] any translation, to the extent that it differs from its source text, may contribute to an analysis of what constitutes the unique character of a literary work» (2006, p. 169). Pour sa part, Fabienne Durand-Bogaert, s'inspirant justement des propos de Peter Szendy, a déjà avancé "qu'un traducteur est à la fois un lecteur qui signe et écrit sa lecture et un auditeur qui signe et écrit son écoute» (2009, p. 30; nous soulignons). Toutefois, aucun de ces traductologues n'approfondit vraiment sa réflexion à cet égard. 
Paradoxalement, c'est encore le musicologue Szendy qui, selon nous, situe le mieux le traducteur littéraire dans le paradigme musical. Dans son livre Listen: A History of Our Ears (2008), il compare explicitement les arrangeurs aux traducteurs littéraires. Pour justifier son analogie, il invoque la nécessité, pour l'un comme pour l'autre, de donner corps à son interprétation (écoute ou lecture) par le biais d'un nouvel écrit (ibid., p. 36), puis d'y apposer sa signature (2001, p. 35). Ce faisant, il remonte à une époque où l'arrangeur jouissait d'une reconnaissance comparable à celui du compositeur, et il va même jusqu'à laisser entendre que l'analogie traductionnelle aurait pu contribuer à la dévaluation du statut de l'arrangeur (2008). Fait intéressant, c'est justement en citant $\mathrm{La}$ tâche du traducteur de Walter Benjamin que Szendy redonne ses lettres de noblesse à la transcription musicale et, par le fait même, à la traduction littéraire. Selon Szendy, l'arrangement comme la traduction produisent des «œuvres originales» qui ne remplacent pas l'œuvre source, mais coexistent avec elle, sur un pied d'égalité (ibid., p. 61). Adaptant les mots de Szendy, nous pourrions donc affirmer que la traduction poétique dépasse la lecture en ceci qu'elle constitue «une écriture de l'écoute. Une écoute qui ne se contenterait pas de recevoir ou percevoir les œuvres, mais qui les incorporerait, qui ferait, à travers un nouveau corps [...], l'expérience de leur résistance» (2001, p. 15). À l'inverse de ce que déclare Derrida (1982, p. 202), la traduction poétique pourrait donc effectivement constituer une "transcription", mais au sens musical. Cela dit, au terme de la discussion, nous serions plutôt tentée de moduler les propos de Fabienne Durand-Bogaert (2009) pour en refocaliser la métaphore: le traducteur de poésie n'est ni vraiment un lecteur ni un arrangeur qui écrit, mais plutôt un écrivain, un créateur à part entière qui signe sa lecture et son écoute du texte d'un autre.

\section{Références}

Bantaş, Andrei (1989). "A Few Hypotheses on Translating Poetry». Revue roumaine de linguistique, 34, 2, p. 147-171.

Berman, Antoine (1984). L'épreuve de l'étranger - culture et traduction dans l'Allemagne romantique. Paris, Gallimard.

Bly, Robert (1983). The Eight Stages of Translation. Boston, Rowan Tree Press.

Bougault, Laurence (2005). «Illisibilité ou polysémie généralisée dans la poésie moderne?» In Olivier Soutet, dir. La Polysémie. Paris, Presses de l'Université Paris-Sorbonne, p. 439-450. 
Brault, Jacques (1989). «Sur la traduction de la poésie (1977)». La poussière du chemin. Montréal, Boréal, p. 201-215.

Caillois, Roger (1978). «Postface». In J. Berque et al., dir. Colloque sur la traduction poétique. Centre Afrique-Asie-Europe de la Sorbonne nouvelle, décembre 1972. Paris, Gallimard, p. 305-312.

Davoust, André (1994). «Traductologie et poésie: Emily Dickinson entre la dérive des règles et les règles de la dérive». Cahiers Charles $V, 17$, p. 111-186.

De Beaugrande, Robert (1978). Factors in a Theory of Poetic Translating. Assen, Van Gorcum.

Derrida, Jacques (1982). L'oreille de l'autre: otobiographies, transferts, traductions - Textes et débats avec Jacques Derrida. Sous la direction de C. Lévesque et C.V. Macdonald. Montréal, VLB.

Durand-Bogaert, Fabienne (2009). "Oublier l'image, tendre l'oreille». Nouvelle revue esthétique, 3, p. 27-30.

Dussart, André (1994). "L'empathie, esquisse d'une théorie de la réception en traduction». Meta, 39, 1, p. 107-115.

Eco, Umberto (1972). La structure absente - Introduction à la recherche sémiotique. Paris, Mercure de France.

Eco, Umberto (1985). Lector in fabula ou la Coopération interprétative dans les textes narratifs. Trad. Myriam Bouzaher. Paris, Grasset.

Eco, Umberto (1992). Les limites de l'interprétation. Trad. Myriam Bouzaher. Paris, Grasset.

Eco, Umberto (1996). Interprétation et surinterprétation. Paris, PUF, coll. «Formes sémiotiques».

Eco, Umberto (2001). Experiences in Translation. Trad. Alastair McEwen. Toronto, University of Toronto Press.

Folkart, Barbara (1999). «Poetry as Knowing». TTR, 12, 1, p. 31-55.

François, Rose-Marie (1999). «Dans l'atelier du traducteur de poèmes». Les cabiers internationaux de symbolisme. Théorie et pratique de la traduction III, 92-94, p. 73-83.

Gadamer, Hans Georg (1982). L'art de comprendre - Écrits 2: Herméneutique et champs de l'expérience humaine. Paris, Aubier, p. 139-239.

Golden, Seán (1997). “'Whose Morsel of Lips Will you Bite?' Some Reflections on the Role of Prosody and Genre as Non-Verbal Elements in the Translation of Poetry». In F. Poyatos, dir. Nonverbal Communication and Translation - Nerw Perspectives and Challenges in Literature, Interpretation and the Media. Amsterdam/Philadelphie, John Benjamins, p. 217-245. 
Green, Jeffrey M. (2001). «Metaphors for Translation». Thinking Through Translation. Athens, GA, University of Georgia Press, p. 6-21.

Jones, Francis R. (1989). «On Aboriginal Sufferences: A Process Model of Poetic Translating». Target, 1, 2, p. 183-199.

Jones, Francis R. (2006). «Unlocking the Black Box: Researching PoetryTtranslation Processes». In E. Loffredo et M. Perteghella, dir. Translation and Creativity - Perspectives on Creative Writing and Translation Studies. London, Continuum, p. 59-74.

Kayra, Erol (1998). «Le langage, la poésie et la traduction poétique ou une approche scientifique de la traduction poétique». Meta, 43, 2, p. 1-8.

Maisonneuve, Roland (1978). «La musique du mot et du concept, ou certains problèmes de traduction poétique». Meta, 23, 1, p. 73-85.

Millis, Christopher (1988). "The Three Stages of Translation: Translating a Poem by Umberto Saba». Translation Review, 37, p. 17-24.

Nicolas, François (2000). «La puissance et la gloire de la transcription (De la confrontation Schoenbert-Busoni)». Arrangements, dérangements: la transcription musicale aujourd'hui. Textes réunis par P. Szendy. Montréal, Ircam, p. 45-60.

Ricœur, Paul (1986). Du texte à l'action - Essais d'herméneutique II. Paris, Éditions du Seuil, coll. «Esprit».

Ricœur, Paul (1991). A Riccur Reader: Reflection and Imagination. Mario J. Valdés, dir. Toronto, University of Toronto Press.

Ricœur, Paul (2004). Sur la traduction. Paris, Bayard.

Scott, Clive (2006). «Translating the Literary: Genetic Criticism, Text Theory and Poetry». In S. Bassnett et P. Bush, dir. The Translator as Writer. London et New York, Continuum, p. 106-118.

Steiner, George (1978). On Difficulty and Other Essays. Oxford, Oxford University Press.

Steiner, George (1988). Le sens du sens - Présences réelles, Real Presences, Realpräsenz. Trad. française Monique Philonenko, trad. allemande Heinz Wismann. Paris, Librairie philosophique J. Vrin.

Steiner, George (1992 [1975]). After Babel - Aspects of Language and Translation. Oxford, Oxford University Press,

Steiner, George (2003). Extraterritorialité - Essais sur la littérature et la révolution du langage. Trad. Pierre-Emmanuel Dauzat. Paris, Hachette, coll. «Littératures».

Stratford, Madeleine (2011). «Un poème de Pizarnik sous toutes ses coutures: vers une nouvelle méthode d'analyse des traductions poétiques». TTR, 24, 1, p. 143-176. 
Szendy, Peter (2000). "L'arrangement dérange...» Arrangements, dérangements: la transcription musicale aujourd'bui. Textes réunis par P. Szendy. Montréal, Ircam, p. 7-15.

Szendy, Peter (2008). Listen: A History of Our Ears. New York, Fordham University Press.

Turner, Allan (2006). «Translation and Criticism: The Stylistic Mirror ». Yearbook of English Studies, 36, 1, p. 168-176.

Verhesen, Fernand (2003). À la lisière des mots - Sur la traduction poétique. Bruxelles, Éditions de La Lettre volée, «coll. Palimpsestes».

Vigneault, Érik (1999). «Herméneutique et traduction poétique: quelques remarques». TTR, 12,2, p. 173-188.

Wittbrodt, Andreas (1995). Verfahren der Gedichtiibersetzung. Frankfurt am Main, Peter Lang.

Wuilmart, Françoise (1999). «Le péché de 'nivellement' dans la traduction littéraire». Les cabiers internationaux de symbolisme. Théorie et pratique de la traduction, III, 92-94, p. 213-224.

Zhang, Jian (1997). «Reading Transaction in Translation». Babel, 43, 3, p. 237-250.

Madeleine Stratford Université du Québec en Outaouais Département d'études langagières 283, boulevard Alexandre-Taché, bureau F-1052 Gatineau (Québec) J8X 3X7 CANADA madeleine.stratford@uqo.ca 


\section{De compte-fil à garde-fou}

\section{Annexe}

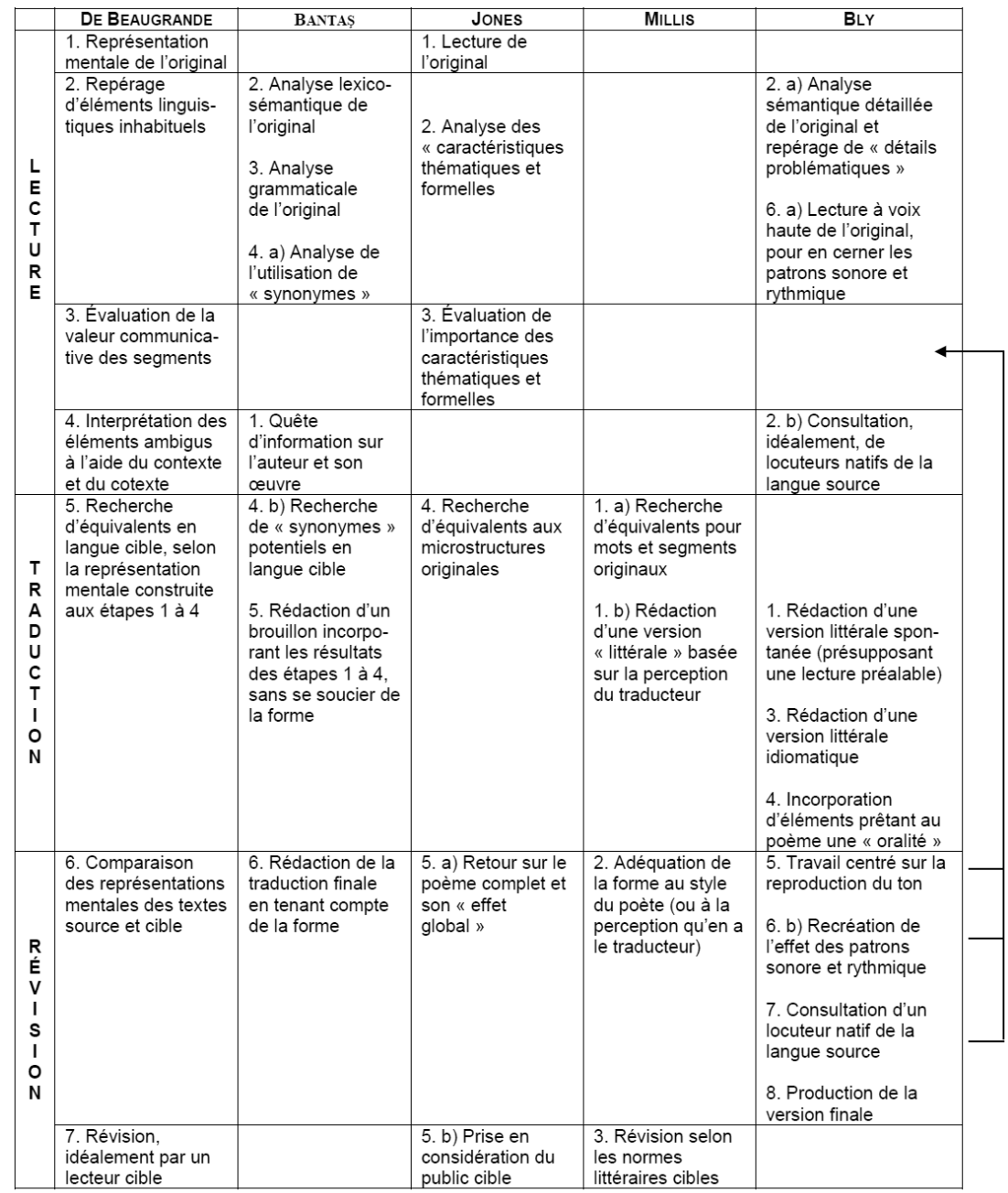

